\title{
RELACIONES ENTRE APOYO PARENTAL, AUTOEFICACIA Y ACTIVIDAD FÍSICA EN ADOLESCENTES
}

\author{
Manuel J. de la Torre-Cruz \\ Departamento de Psicología (Universidad de Jaén) \\ E-mail: majecruz@ujaen.es
}

https://doi.org/10.17060/ijodaep.2017.n1.v2.959

Fecha de Recepción: 9 Marzo 2017

Fecha de Admisión: 1 Abril 2017

\section{RESUMEN}

Este estudio examinó las relaciones existentes entre el apoyo parental hacia la actividad física, la frecuencia de práctica semanal y las expectativas de autoeficacia para superar los obstáculos que dificultan dicha participación. Asimismo, se analizó si la frecuencia de práctica y autoeficacia percibida difería en función del sexo del participante. Un total de 430 estudiantes procedentes de tres centros de Educación Secundaria Obligatoria de la provincia de Jaén, con edades comprendidas entre los 12 y 17 años de edad ( $M=13.96$; $S D=1.39$ ) tomaron parte en este estudio. Los resultados mostraron que los chicos y chicas que atribuían a sus progenitores un elevado nivel de apoyo instrumental, modelado y limitación de la conducta destinaban un mayor número de días a la semana a la realización de actividad físico-deportiva durante un intervalo mínimo de 60 minutos y se mostraban más autoeficaces para implicarse en dicha práctica en comparación con aquellos otros que percibían en sus progenitores menores índices de apoyo. Asimismo, tanto la frecuencia de práctica de actividad como la autoeficacia atribuida para implicarse en actividades de naturaleza físico-deportiva fue mayor en el caso de los participantes varones.

Palabras clave: apoyo parental, adolescentes, autoeficacia y actividad física

\section{ABSTRACT}

This study examined the relationships between parental support for physical activity, the frequency of weekly practice, and expectations of self-efficacy to overcome obstacles that impede such participation. We also analyzed whether the frequency of practice and perceived self-efficacy were different according to the participant's gender. A total of 430 students, from three secondary education centers in the province of Jaen and aged between 12 and 17 years $(M=13.96$; $S D=1.39)$, took part in this study. The results showed that the boys and girls who attributed to their parents a high level of instrumental support, modeling, and behavioral limitation allocated a greater number of days a week to the performance of physical-sport activity during a minimum interval of 60 min- 


\section{RELACIONES ENTRE APOYO PARENTAL, AUTOEFICACIA Y ACTIVIDAD FÍSICA EN ADOLESCENTES}

utes. In addiction, these were more self-efficacious to engage in such practice compared to those who perceived lower support rates in their parents. Finally, both the frequency of practice of activity and the self-efficacy attributed to being involved in activities of a physical-sport nature was greater in the case of male participants.

Key words: parental support, adolescents, self-efficacy and physical activity

\section{INTRODUCCIÓN}

La práctica de actividad física junto a la disminución de la frecuencia de conductas sedentarias mantiene una relación positiva con la salud física y psicológica de niños y adolescentes (Ho, Louie, Chow, Wong e Ip, 2015; Moljord, Moksnes, Espnes, Hjemdal y Eriksen, 2014; Sheperd, Krägeloh, Ryan y Schofield, 2012). A pesar de los beneficios el porcentaje de jóvenes que satisface el criterio de realización de actividad físico-deportiva a una intensidad que oscila entre moderada y vigorosa por un período de 60 minutos durante al menos cinco días a la semana ha disminuido en las dos últimas décadas (Davison, Li, Baskin, Cox y Affuso, 2010; Graham, Bauer, Friend, Barr-Anderson y Neumark-Sztainer, 2014; Lee, Nihiser, Strouse, Das, Michael y Huhman, 2010).

La teoría cognitivo-social de Bandura (1997) sugiere que la provisión de apoyo social incrementa la autoeficacia de los individuos para superar los obstáculos que dificultan la posibilidad de ser físicamente activos. Una de las fuentes principales de apoyo se encuentra en el ámbito familiar. Madres y padres, a través de ayudas tangibles (aportando el equipamiento necesario o trasladando a sus hijos hasta los lugares de práctica) e intangibles (exhibiendo muestras de ánimo y felicitando a los hijos por su participación), condicionan la frecuencia e intensidad de las conductas asociadas a la práctica de actividad física en niños y adolescentes (Laird, Fawkner, Kelly, McNamee y Niven, 2016; Peterson, Lawman, Fairchild, Wilson y van Horn, 2013; Trost and Loprinzi, 2011).

La investigación indica que tanto el modelado como el apoyo logístico parental contribuyen al mantenimiento de la frecuencia de actividad física de jóvenes adolescentes (Davison y Jago, 2009). Asimismo, Verloigne y cols. (2014), constataron que la autoeficacia percibida para la práctica de actividad física (implicarse en su realización pese a las restricciones temporales) mediaba la relación entre el apoyo parental y la actividad física de niños y adolescentes durante los días laborables y los fines de semana. Por su parte, Sebire, Haase, Montgomery, McNeill y Jago (2014) obtuvieron que tanto el modelado como el apoyo instrumental materno junto al modelado paterno predecían de forma positiva la autoeficacia percibida para implicarse en dicha práctica.

Sebire y cols. (2014), plantean que una limitación de los estudios que examinan los correlatos psicosociales de las prácticas parentales relativas a la actividad física estriba en el hecho de ignorar la posible influencia de las prácticas tanto maternas como paternas. La investigación sugiere que las chicas pueden recibir o percibir diferentes grados de apoyo por parte de madres y padres e incluso existir diferencias evolutivas en el apoyo recibido (Davison, Cutting y Birch. 2003; Davison y Jago, 2009).

Asimismo, la investigación previa ha considerado al apoyo parental en términos genéricos, a saber, como una combinación de diferentes formas del mismo. Dado que éstos distintos tipos (logístico, modelado, establecimiento de límites) podrían incidir diferencialmente la práctica de actividad física, el examen de la influencia individual de cada uno de ellos podría esclarecer aún más estas relaciones.

Así, el objetivo de este estudio residió, de un lado, en el examen de las relaciones existentes entre apoyo instrumental, modelado y límites parentales a la conducta sedentaria, frecuencia semanal de actividad física y expectativas de autoeficacia para superar los obstáculos que dificultan dicha participación. Adicionalmente se exploró si la cantidad de práctica y autoeficacia percibida difería en 
función del sexo del participante. Baptista y cols. (2012) encontraron diferencias favorables a los varones de entre 10 y 15 años de edad en la cantidad de actividad física ligera, moderada y vigorosa, evaluada mediante procedimientos de acelerometría. Asimismo, Li, lannotti, Haynie, Perlus y Simons-Morton (2014), obtuvieron que el porcentaje de chicos adolescentes considerados físicamente activos (al menos cinco horas de práctica semanal) estaba próximo a duplicar al de chicas activas $(62 \%$ vs. $37 \%)$.

Se hipotetizó que la percepción de un mayor apoyo parental se asociaría con una mayor autoeficacia percibida para la práctica de actividad física junto a una mayor frecuencia semanal de práctica de intensidad moderada a vigorosa autoinformada. A su vez, se estableció que tanto la autoeficacia como la frecuencia de práctica serían más elevadas en el caso de los varones.

\section{MÉTODO}

\section{Participantes}

Un total de 430 estudiantes procedentes de tres centros de Educación Secundaria Obligatoria de la provincia de Jaén, con edades comprendidas entre los 12 y 17 años de edad ( $M=13.96$; $S D=$ 1.39) tomaron parte en este estudio. El número de grupos-clase evaluados ascendió a un total de 22. La distribución en cuanto a la variable sexo fue ligeramente superior en el caso de las chicas (n $=225,52.3 \%$ ).

\section{Instrumentos}

Para evaluar la percepción de apoyo parental se utilizó una versión adaptada al castellano de la "Activity Support Scale, ACTS" propuesta por Davison (2004). Esta escala está compuesta por nueve ítems agrupados en tres factores que reciben la siguiente denominación: apoyo instrumental ("Me llevó a lugares donde podía realizar actividad física"), modelado ("Me animó a ser físicamente activo/a actuando como ejemplo y siendo modelo a imitar") y establecimiento de límites a la conducta sedentaria ("Me limitó la cantidad diaria de horas que podía dedicar a jugar con los videojuegos"). Los participantes debían de indicar a lo largo de una escala tipo Likert de cuatro puntos el grado de acuerdo ( 1 = total desacuerdo, 4 = total acuerdo) con el que, a lo largo del último año, las actuaciones de madres y padres se correspondían con las afirmaciones indicadas. Una mayor puntuación se correspondía con un mayor nivel de apoyo y establecimiento de límites a la conducta sedentaria. Los índices de fiabilidad obtenidos para esta muestra de estudio fueron .71 para la dimensión apoyo instrumental, .74 para la dimensión modelado y .77 para la dimensión límite a la conducta sedentaria.

La escala MVPA "Moderate-to-Vigorous Physical Activity" (Prochaska, Sallis y Long, 2001) informa del número de días durante la semana previa y una semana cualquiera en los que los participantes realizaron actividad física de intensidad moderada a vigorosa durante al menos 60 minutos. La medida define la actividad física de una forma genérica como "cualquier actividad que incrementa la tasa cardíaca y en ocasiones te dificulta respirar". El promedio de los dos ítems produce un valor del número de días a la semana que los adolescentes acumulan 60 minutos de actividad física de intensidad moderada a vigorosa. Un valor de cinco o superior se corresponde con la inclusión del joven en la categoría físicamente activo. El índice de fiabilidad de los dos ítems en la muestra de estudio ascendió a .81 .

La autoeficacia para la práctica de actividad física fue evaluada empleando un cuestionario de ocho ítems desarrollado por Dishman y cols. (2010). Algunos ejemplos de los ítems incluidos fueron: "Soy capaz de realizar actividad física en mi tiempo libre sin importar lo ocupado o ajetreado del día" y "Soy capaz de pedir a mis padres u otros adultos que realicen actividad física conmigo 


\section{RELACIONES ENTRE APOYO PARENTAL, AUTOEFICACIA Y ACTIVIDAD FÍSICA EN ADOLESCENTES}

durante mi tiempo libre". Los ítems fueron valorados a lo largo de una escala tipo Likert en la que el participante debía indicar su grado de acuerdo con las distintas afirmaciones. El rango de valores osciló desde 1 (totalmente en desacuerdo) hasta 5 (totalmente de acuerdo). Se obtuvo un único factor que explicó el $47 \%$ de la varianza de las puntuaciones obtenidas. Una mayor puntuación era equivalente a un mayor nivel de autoeficacia percibida. El índice de fiabilidad para esta muestra de estudio ascendió a .83.

\section{Procedimiento}

Se redactó una carta dirigida a los equipos directivos de dos centros educativos de la provincia de Jaén solicitando su colaboración para materializar la aplicación de pruebas. En la carta se hacía explícito que el objetivo principal del estudio residía en el análisis de las posibles relaciones existentes entre el apoyo que chicos y chicas afirmaban recibir por parte de sus progenitores para la práctica de actividad físico-deportiva y diversas variables relacionadas con dicha participación (frecuencia, disfrute, autoeficacia percibida para la práctica o capacidad para eliminar barreras u obstáculos que dificultan su realización).

Padres o tutores legales debían devolver, convenientemente firmado, un consentimiento que autorizaba la participación del menor en el estudio. Los diferentes cuestionarios se aplicaron durante las horas lectivas destinadas a la asignatura de Educación Física. Los participantes dispusieron de 50 minutos para completar las diferentes medidas. El procedimiento de obtención de datos tuvo en cuenta la normativa legal vigente española que regula la investigación clínica en humanos (Real Decreto 561/1993 sobre ensayos clínicos), la ley de protección de datos de carácter personal (Ley Orgánica 15/1999) y los principios fundamentales establecidos en la Declaración de Helsinki (revisión de 2008).

\section{RESULTADOS}

Atendiendo a las consideraciones anteriores se examinó de forma previa a cualquier otro análisis la posible existencia de diferencias en el grado de apoyo materno y paterno percibido en función del sexo del participante. Al no observarse diferencias estadísticamente significativas se optó por computar una única puntuación para cada dimensión (apoyo instrumental, modelado y establecimiento de límites a la conducta sedentaria) promediando las puntuaciones que chicas y chicos concedieron a madres y padres.

Este hecho supuso que solo se consideraran para posteriores análisis aquellos participantes que aportaran información sobre ambos progenitores. Seguidamente se calculó el valor de la mediana en las diferentes variables (8.50 para apoyo instrumental, 9.00 para modelado y 8.00 para limitaciones a la conducta sedentaria). De este modo, cuando la puntuación alcanzada en cualquiera de ellas era inferior a su respectiva mediana el participante quedaba incluido en la condición bajo apoyo instrumental, bajo modelado y baja limitación a la conducta sedentaria. En aquellos casos en los que la puntuación era superior a la mediana el encuestado formaba parte del grupo de alto apoyo instrumental, alto modelado y alta limitación a la conducta sedentaria.

Relación entre apoyo parental y frecuencia de actividad física.

Se realizaron tres análisis univariados de covarianza (ANCOVAs) con el propósito de conocer la relación existente entre el apoyo familiar percibido para la práctica de actividad físico-deportiva (evaluado mediante el apoyo instrumental, el modelado y la limitación de conductas sedentarias) y la frecuencia informada de práctica semanal de actividad física de intensidad moderada a vigorosa. Asimismo, se intentó determinar si esta relación podía diferir en función del sexo del participante. Dado que la investigación previa revela que el índice de masa corporal y la edad cronológica man- 
tienen una relación negativa con el disfrute y frecuencia de práctica de actividad física éstos dos últimos factores fueron incluidos como covariables.

El primero de los análisis realizado reveló un efecto principal estadísticamente significativo del apoyo parental instrumental, $F(1,395)=51.20, p<.001, \eta^{2}=.12$ y del sexo del participante, $F(1$, $395)=26.81, p<.001, \eta^{2}=.06$. La interacción apoyo instrumental $x$ sexo resultó estadísticamente significativa, $F(1,395)=4.91, p<.05, \eta^{2}=.01$. El análisis de la interacción reveló diferencias estadísticamente significativas en la frecuencia semanal de actividad física realizada por las chicas y chicos que atribuían tanto un bajo, $t(201)=-2.24, p<.001(M=2.47$ vs $M=2.90)$, como elevado apoyo familiar, $t(197)=-4.91, p<.001(M=3.19$ vs $M=4.24)$, así como diferencias dentro de cada uno de los grupos en función del nivel de apoyo percibido, $t(209)=-3.83, p<.001(M=2.47$ vs. $M=3.19)$, en el caso de las chicas y, $t(189)=-6.20, p<.001(M=2.90$ vs $M=4.24)$ en el caso de los chicos. En el gráfico número 1 se representa esta interacción.

Gráfico número 1:

Valores promedios de práctica semanal de actividad físico-deportiva de intensidad moderada a vigorosa en función del apoyo instrumental atribuido a los padres y sel sexo del adolescente.

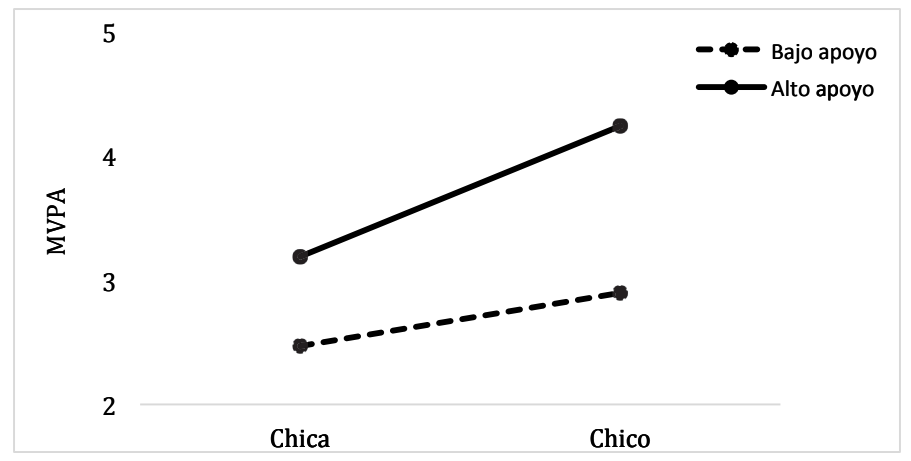

Respecto a la segunda dimensión se observó la existencia de un efecto principal estadísticamente significativo del modelado parental, $F(1,371)=23.52, p<.001, \eta^{2}=.06$, así como del sexo de participante, $F(1,371)=18.48, p<.001, \eta^{2}=.05$. La interacción modelado x sexo no resultó estadísticamente significativa, $F(1,371)=.14, p>.05$. De este modo, los participantes que atribuían una mayor práctica de actividad físico-deportiva a sus progenitores mostraban una mayor frecuencia de actividad práctica semanal ( $M=3.63$ vs $M=2.83$ ). Asimismo, los chicos se implicaban con mayor frecuencia que las chicas en este tipo de actividades ( $M=3.60$ vs $M=2.84$ ).

Finalmente, el tercero de los análisis reveló la existencia de un efecto principal estadísticamente significativo de la variable límites a la conducta sedentaria, $F(1,404)=7.69, p<.01, \eta^{2}=.02$, así como de la variable sexo, $F(1,404)=27.82, p<.001, \eta^{2}=.06$. La interacción límites $x$ sexo no resultó estadísticamente significativa, $F(1,404)=.07, p>$.05. Como en los casos anteriores, la frecuencia de práctica de actividad física fue más elevada en los jóvenes que atribuían a sus progenitores una mayor restricción al comportamiento sedentario ( $M=3.40$ vs $M=2.98)$ y en los chicos frente a las chicas ( $M=3.58$ vs $M=2.79)$.

Relación entre apoyo parental y autoeficacia percibida para la práctica de actividad física.

El primero de los análisis realizado reveló un efecto principal estadísticamente significativo del 


\section{RELACIONES ENTRE APOYO PARENTAL, AUTOEFICACIA Y ACTIVIDAD FÍSICA EN ADOLESCENTES}

apoyo parental instrumental, $F(1,406)=82.33, p<.001, \eta^{2}=.17$ y del sexo del participante, $F(1$, $406)=6.13, p<.05, \eta^{2}=.02$. La interacción apoyo instrumental $x$ sexo no resultó estadísticamente significativa, $F(1,406)=.08, p>.05$. Concretamente, las mayores puntuaciones en autoeficacia percibida para la realización de actividad física se observaron en aquellos participantes que atribuyeron a sus padres un elevado grado de apoyo instrumental ( $M=29.40$ vs $M=23.06$ ).

El segundo de los análisis arrojó la existencia de un efecto principal estadísticamente significativo de la variable modelado, $F(1,383)=46.28, p<.001, \eta^{2}=.11$. Ni el sexo, $F(1,383)=3.64, p>$ .05 , ni la interacción modelado x sexo, $F(1,383)=.01, p>.05$, resultaron estadísticamente significativas. Los adolescentes que consideraban que tanto madres como padres eran modelos frecuentes de actividad física a los que imitar informaron de mayores expectativas de autoeficacia para la práctica de actividad física $(M=29.04$ vs $M=23.71)$.

Finalmente, el último de los análisis realizados mostró la existencia de un efecto principal estadísticamente significativo de la limitación parental de las conductas sedentarias, $F(1,405)=13.64$, $p<.001, \eta^{2}=.03$, así como del sexo del participante, $F(1,405)=5.27, p<.05$. La interacción establecimiento de límites $x$ sexo no alcanzó la significatividad estadística, $F(1,405)=.11, p>.05$. En este caso, la autoeficacia percibida para la práctica de actividad física fue mayor en el grupo de adolescentes que percibían en sus progenitores una mayor imposición de límites a la realización de conductas sedentarias ( $M=27.69$ vs $M=24.82$ ). Asimismo, los chicos se atribuyeron una mayor autoeficacia para la práctica de actividad física de lo que lo hicieron las chicas, $(M=27.00$ vs $M=25.01)$.

\section{DISCUSIÓN Y CONCLUSIONES}

El apoyo familiar percibido para la práctica de actividad físico-deportiva se relaciona de forma positiva con la frecuencia de práctica semanal y la autoeficacia percibida para superar las barreras u obstáculos que dificultan o imposibilitan la realización de la citada práctica en un grupo de jóvenes adolescentes. Estos resultados son coincidentes con los arrojados por investigaciones precedentes (Sebire y cols. 2014; Trost y Loprinzi, 2011; Verloigne y cols. 2014).

Los jóvenes que atribuían a sus progenitores un elevado nivel de apoyo instrumental destinaban un mayor número de días a la semana a la realización de actividad físico-deportiva durante un intervalo mínimo de 60 minutos en comparación con aquellos otros que percibían en sus progenitores un menor apoyo instrumental.

Estos resultados son coincidentes con los obtenidos por Timperio y cols. (2013) en cuyo estudio tanto la disposición de equipamiento deportivo en el hogar como la aportación económica de los padres y el apoyo logístico (transportando al menor hasta los lugares de práctica) mostraban relaciones positivas con la frecuencia semanal con la que chicos y chicas participaban en actividades deportivas durante un período mínimo de 30 minutos. No obstante, esta asociación estuvo parcialmente mediada por las cogniciones de los jóvenes. Así, la influencia del ambiente familiar sobre la cantidad de práctica deportiva incidía de forma indirecta a través de las actitudes, creencias, expectativas de autoeficacia y diversión que suscitaba dicha participación.

Asimismo, los participantes que consideraron a sus progenitores un mayor modelado de conductas asociadas a la práctica de actividad física expresaron ser más activos y más autoeficaces con relación a sus iguales cuyo modelado parental atribuido fue menor. En sintonía con este hallazgo, Jago y cols. (2014) examinaron la relación existente entre la actividad física semanal que padres e hijos (entre cinco y seis años de edad) habían realizado mediante el registro de acelerómetros. Los resultados revelaron que esta forma de modelado participante arrojó una relación positiva, aunque débil, entre la práctica de actividad física desplegada por padres e hijos. Sin embargo, la citada relación fue mucho más elevada cuando los padres fueron divididos entre quienes satisfacían las reco- 
mendaciones internacionales de práctica de actividad física diaria (al menos 30 minutos a una intensidad que oscila entre moderada y vigorosa) y los que no.

En una línea similar, Jacoby y cols. (2011), analizaron la actividad física de la unidad familiar mediante el empleo de podómetros calculando la correlación entre el número de pasos que padres e hijos realizaban diariamente a lo largo de una semana. Los resultados obtenidos revelaron la existencia de correlaciones positivas y significativas en las díadas madre-hija. Asimismo, el valor de la correlación incrementaba conforme lo hacía la edad del hijo, siendo dicho incremento mayor en las chicas.

Finalmente, la percepción de una mayor imposición familiar de límites a la realización de actividades 0 conductas sedentarias se asoció de forma positiva con una mayor frecuencia de práctica semanal de actividad física y autoeficacia percibida para dicha práctica. Resulta de este modo crucial la necesidad de elaborar e implementar programas de intervención que incorporen a madres y padres como fuentes primordiales de apoyo social destinadas a la modificación de conductas asociadas a la actividad física de niños y adolescentes.

De otro lado, en la práctica totalidad de análisis realizados tanto la frecuencia de práctica de actividad como la autoeficacia atribuida para implicarse en actividades de naturaleza físico-deportiva fue mayor en el caso de los participantes varones, confirmándose así los hallazgos obtenidos en investigaciones previas (Baptista y cols., 2012; Li y cols., 2014). Estos resultados sugieren que las propuestas de intervención deberían instar a que la promoción de apoyos familiares fuese especialmente notoria en el caso de las chicas por ser consideradas un grupo de riesgo tendente hacia un estilo de vida más sedentario.

A pesar de los resultados obtenidos este estudio presenta algunas limitaciones. El empleo de un diseño transversal excluye la posibilidad de establecer conclusiones sobre la direccionalidad de las relaciones observadas. Las teorías que abogan por la reciprocidad de las relaciones interpersonales (por ejemplo, la influencia mutua que se tiene lugar entre padres e hijos) postulan que tales dinámicas padre-hijo operan en un ciclo permanente de influencia y cambio. Por tanto, cabe la posibilidad de que los procesos de socialización analizados en este estudio tengan una naturaleza bidireccional donde las prácticas parentales asociadas a la actividad física incidan sobre las actitudes y conductas de los hijos al tiempo que éstos últimos ofrezcan ciertas claves a sus progenitores que regulen los tipos y niveles de actuaciones familiares destinadas a la promoción de la participación en actividades de naturaleza físico-deportiva. Asimismo, cabe la posibilidad de que estos procesos evolucionen con el paso del tiempo y supongan cambios paulatinos en la vida de chicas y chicos. De otro lado, el uso de medidas de autoinforme supone una limitación añadida a este estudio. Evaluar las posibles asociaciones mediante la inclusión de medidas objetivas de actividad física (por ejemplo, recurriendo al uso de la acelerometría) podría convertirse en una interesante vía de investigación.

\section{REFERENCIAS BIBLIOGRÁFICAS}

Bandura, A. (1997). Self-efficacy: The exercise of control. New York: Freeman.

Baptista, F., Santos, D. A., Silva, A. M., Mota, J., Santos, R., Vale, S. y cols. (2012). Prevalence of the Portuguese population attaining sufficient physical activity. Medicine \& Science in Sports \& Exercise, 44, 466-473.

Davison, K. K. y Deane, G. D. (2010). The consequence of encouraging girls to be active for weight Ioss. Social Science Medicine, 70, 518-525.

Davison, K. K. (2004). Activity-related support from parents, peers and siblings and adolescents physical activity: Are there gender differences? Journal of Physical Activity and Health, 1, 363- 
376.

Davison, K. K., Cutting, T. M. y Birch, L. L. (2003). Parents activity-related parenting practices predict girls physical activity. Medicine Science and Sports Exercise, 35, 1589-1595.

Davison, K. K. y Jago, R. (2009). Change in parent and peer support across ages 9 to 15 years and adolescent girls physical activity. Medicine \& Science in Sports and Exercise, 41, 1816-1825.

Dishman, R. K., Hales, D. P., Sallis, J. F., Saunders, R., Dunn, A. L., Bedimo-Rung, A. L. y Ring, K. B. (2010). Validity of social-cognitive measures for physical activity in middle-school girls. Journal of Pediatric Psychology, 35, 72-88. Doi:10.1093/pepsy/jsp031

Graham, D. J., Bauer, K. W., Friend, S., Barr-Anderson, D. J. y Neumark-Sztainer, D. (2014). Personal, behavioral, and socio-environmental correlates of physical activity among adolescent girls: Cross-sectional and longitudinal associations. Journal of Physical Activity and Health, 11, 51-61.

Ho, F. K. W., Louie, L. H. T., Chow, C. B., Wong, W. H. S. e Ip, P. (2015). Physical activity improves mental health through resilience in Hong Kong Chinese adolescents. BMC Pediatrics, 15:48. Doi: 10.1186/212887-015-0365-0

Jacobi, D., Caille, A., Borys, J. M., Lommez, A., Couet, C., Charls, M. A., Oppert, J. M. y FLVS Study Group (2011). Parent-offspring correlations in pedometer-assessed physical activity. Plos One, 6, e29195. Doi:10.1371/journal.pone.0029195

Jago, J., Thompson, J. L., Sebire, S. J., Wood, L., Pool, L. Zahra, J. y Lawlor, D. A. (2014). Crosssectional associations between the screen-time of parents and young children: Differences by parent and child gender and day of the week. International Journal of Behavioral Nutrition and Physical Activity, 11:54. Doi:10.1186/1479-5868-11-54

Laird, Y., Fawkner, A., Kelly, P., McNamee, L. y Niven, A. (2016). The role of social support on physical activity behavior in adolescent girls: a systematic review and meta-analysis. International Journal of Behavioral Nutrition and Physical Activity, 13:79. Doi: 10.1186/s12966-016-0405-7

Lee, S. M., Nihiser, A., Strouse, D., Das, B., Michael, S. y Huhman, M. (2010). Correlates of children and parents being physically active together. Journal of Physical Activity and Health, 7, 776-783.

Li, K., Iannotti, R. J., Haynie, D. L., Perlus, J. G. y Simons-Morton, B. G. (2014). Motivation and planning as mediators of the relation between social support and physical activity among U.S. adolescents: a nationally representative study. International Journal of Behavioral Nutrition and Physical Activity, 11:42.

Moljord, I. E. O., Moksnes, U. K., Espnes, G. A., Hjemdal, O. y Eriksen, L. (2014). Physical activity, resilience, and depressive symptoms in adolescence. Mental Health and Physical Activity, 7, 7985. Doi:10.1016/j.mhpa.2014.04.001

Peterson, M. S., Lawman, H. G., Wilson, D. K., Fairchild, A. y van Horn, R. (2013). The association of self-efficacy and parent social support on physical activity in male and female adolescents. Health Psychology, 32, 666-674.

Prochaska, J. J., Sallis, J. F. y Long, B. (2001). A physical activity screening measure for use with adolescents and primary care. Archives of Pediatrics and Adolescents Medicine, 155, 554-559.

Sebire, S. J., Haase, A. M., Montgomery, A. A., McNeill, J., y Jago, R. (2014). Associations between physical activity parenting practices and adolescent girls self-perceptions and physical activity intentions. Journal of Physical Activity and Health, 11, 734-740. Doi:10.1123/jpah.2012-0034

Shepherd, D., Krägeloh, C., Ryan, C. y Schofield, G. (2012). Psychological well-being, self-reported physical activity levels, and attitudes to physical activity in a sample of New Zealand adolescent females. Psychology, 3, 447-453.

Timperio, A. F., van Stralen, M. M., Brug, J., Bere, E., Chinapaw, M. J., De Bourdeaudhuij I., Jan, 
N.,... and on behalf of the ENERGY consortium (2013). Direct and indirect associations between the family physical activity environment and sports participation among 10-12 year-old European children: testing the EnRG framework in the ENERGY project. International Journal of Behavioral Nutrition and Physical Activity, 10:15

Trost, S. G. y Loprinzi, P. D. (2011). Parental influences on physical activity behavior in children and adolescents: A brief review. American Journal of Lifestyle Medicine, 5, 171-181.

Verloigne, M., Veitch, J., Carver, A., Salmon, J., Cardon, G., Bourdeaidhuij, I., y Timperio, A. (2014). Exploring associations between parental and peer variables and physical activity among adolescents: A mediation analysis. BMC Public Health, 14:996. 
